\title{
Relación de factores sociodemográficos y habilidades psicolingüís- ticas en estudiantes fronterizos y transfronterizos.
}

\author{
Relationship of demographic factors and psycholinguistic skills in border and \\ cross-border students.
}

Martínez-Siza, Diana-Carolina*1; Carvajal-Villamizar, Yuliana-Mercedes*2; Baron-Torres, Heidy- Julieth³; Pabón-Villamizar, Juan-José*4.

Como citar este artículo: Martínez-Siza, Diana-Carolina; Carvajal-Villamizar, Yuliana-Mercedes; Baron-Torres, Heidy- Julieth; Pabón- Villamizar, Juan-José. Relación de factores sociodemográficos y habilidades psicolingüísticas en estudiantes fronterizos y transfronterizos. Revistas Signos Fónicos. 2019; 5(2):1-17.

Correspondencia autor: dmartinez.siza@gmail.com , (Diana Carolina Martínez Siza)

Recibido: Enero 20, 2019.

Aprobado: Mayo 1, 2019.

\section{RESUMEN}

INTRODUCCIÓN: El objetivo es conocer la influencia de los factores sociodemográficos en las habilidades psicolingüísticas en escolares. MÉTODOS: Constituye un estudio exploratorio, descriptivo, en 1735 escolares, evaluando sus habilidades psicolingüísticas por medio del ITPA-3 y recolectando información de sus factores sociodemográficos; analizando los resultados por medio del software estadístico SPSS V.21 y The R Project for Statistical Computing. RESULTADOS: Se obtuvo una recolección de los datos sociodemográficos y la puntuación de las pruebas, plasmando el análisis gráfico usando el programa R, que mostró las relaciones de edad, grado y tiempo que vive en el país con los componentes lingüísticos y orales. ANÁLISIS Y DISCUSIÒN: Los factores que determinan relación con los procesos psicolingüísticos de manera general con los datos se establecen entre género y edad puesto que representan habilidades que se adquieren desde un principio. CONCLUSIONES: El desarrollo de las habilidades psicolingüísticas constituyen factores influyentes entre la edad y el grado.

PALABRAS CLAVES: Habilidades psicolingüísticas, Demografía, Escolares, Frontera, Rendimiento académico.

\begin{abstract}
INTRODUCTION: The objective is to know the influence of demographic factors on psycholinguistic skills in schoolchildren. METHODS: It constitutes an exploratory study, descriptive, in 1735 schoolchildren, evaluating their psycholinguistic skills through ITPA-3 and gathering information of their demographic factors; Analyzing the results by means of the statistical software SPSS V. 21 and the R Project for statistical Computing. RESULTS: A collection of the demographic data and the scoring of the tests

1 *Fonoaudióloga, dmartinez.siza@gmail.com, Orcid: 0000-0003-3353-8889, Vital IPS Arauca, Colombia.

2 *Fonoaudióloga, rehabilitacion@projectionlife.com, Orcid: 0000-0001-9037-9728, Projection Life SA, Colombia.

3 *Fonoaudióloga, hjbaron06@gmail.com, Orcid: 0000-0003-3989-8789, Optimus ( Centro de Atención integral en salud ), Colombia.

4 *Fonoaudiólogo, juanjosepabonv@gmail.com, Orcid: 0000-0002-3901-7622, Rehabilitación Integral Martha Omaña \& Asosiados, Colombia.
\end{abstract}


was obtained, capturing the graphic analysis using the R program, which showed the relationships of age, degree and time that lives in the country with the linguistic and oral components. ANALYSIS AND DISCUSSION: The factors that determine respect with the psycholinguistic processes in a general way with the data are established between gender and age since they represent skills that are acquired from the beginning. CONCLUSIONS: The development of psycholinguistic skills are influential factors between age and grade.

KEYWORDS: Psycholinguistic Skills, Demography, School, Frontiere, Academic Performance.

\section{INTRODUCCIÓN}

Los procesos psicolingüísticos que los infantes desarrollan en la etapa previa a la escuela resultan muy importantes, pues constituyen la base del aprendizaje (1), así como también, permiten el desarrollo de sus capacidades lingüísticas, cognitivas y socio afectivas. Desde el enfoque psicolingüístico no es posible hablar sobre la lengua escrita si no se tiene en cuenta su relación con el lenguaje oral. El objetivo primordial es llegar al aprendizaje permanente y efectivo que le permita interactuar en diferentes contextos de la vida (1).

Lo anterior, se fundamenta con afirmaciones sobre el aprendizaje de la lectoescritura y el correcto desarrollo del lenguaje oral, tanto a nivel comprensivo y expresivo potenciando así las habilidades lingüísticas.

Algunos de los niños que inician la etapa escolar presentan dificultades en cada uno de los componentes lingüísticos necesarios para el proceso de adquisición de la lectoescritura, abordar ampliamente esta problemática, implica un gran reto, pues son aspectos que hoy en día a pesar del gran avance de las neurociencias, la psicología y la interdisciplinariedad no están totalmente identificados (2).

Una de las disciplinas que se encarga de los factores mencionados anteriormente es la psicolingüística, definida como el estudio de las relaciones entre comunicaciones o mensajes y las características de las personas que comunican, específicamente, se basa en el estudio del lenguaje y el modo en que se relaciona con los usuarios, incluyendo procesos por los cuales un hablante o escritor emite señales o símbolos, así como los procesos por los que estas señales se interpretan (2).

En este orden de ideas, surge la necesidad de evaluar y analizar cada uno de los componentes de la psicolingüística en los escolares y sus dificultades, para cumplir con este objetivo se hace necesaria la implementación de la Prueba Illinois de Habilidades Psicolingüísticas versión 3 (ITPA-3), diseñada por Donald D. Hammill, Nancy Mather y Rhia Roberts. Esta prueba es elaborada con el modelo comunicativo expuesto por Osgood (1957) el cual, categoriza los fenómenos presentados a nivel de input (auditivo-visual) o a nivel de output (auditivo-vocal y visual-motor), el ITPA-3 busca explorar las características cognitivas y lingüísticas únicas de cada niño que ayudarán a explicar las habilidades y discapacidades de aprendizaje(2).

Un factor influyente en las dificultades del aprendizaje es el rendimiento académico, caracterizado por el cumplimiento de los logros establecidos en el grado que está cursando un escolar, en muchos casos, estas falencias se reflejan en la pérdida de asignaturas académicas o deserción; una de las necesidades por la cuales se desarrolla este proyecto se basa en el bajo rendimiento académico de los escolares, es común que el rendimiento escolar vaya ligado con dificultades cognitivas tales como la atención, percepción del habla, memoria de trabajo fonológica y control ejecutivo (3).

Según estudios encontrados en los primeros años de escolaridad se evidencia la persistencia de dificultades en el lenguaje oral relacionándose ésta con la lectoescritura (3). En un estudio realizado se presentan hallazgos significativos en cuanto a la relación de las habilidades psicolingüísticas con el rendimiento académico, pues se halla una asociación de las dificultades en las subpruebas evaluadas, con el bajo rendimiento académico (3). En el escenario donde se desarrolló el proceso investigativo se evidencian informes de resultados individuales que muestran un porcentaje mayor al $50 \%$ que refleja que la población no tiene un buen desempeño escolar (Resultados extraídos del informe académico expuesto en el primer periodo 2018 ), además, se reflejan las inquietudes de los docentes acerca de los problemas académicos de los escolares, refiriendo que la mayor causa de barreras de aprendizaje se dan por apatía y falta de responsabilidades en el escolar.

Se recomienda prestar la debida atención a la influencia de los factores sociodemográficos en la percepción que tienen los sujetos; en esta línea, se han desarrollado investigaciones en las que se evalúan los valores de los sujetos en función de aspectos como género, nivel socioeconómico y nivel educativo (4). Por ello se hace necesario describir el análisis sociodemográfico que abarca aquellas técnicas que indagan acerca de las relaciones entre los factores determinantes y el estado de la población y sus

Revista Científica Signos Fónicos, 2019,5(2): 1-17. ISNN 2422-1716. 
cambios.

Para esta investigación es sumamente importante, conocer cuáles son los factores sociodemográficos y cómo se desenvuelven en un contexto, puesto que los mismos, estudian las características de una población y su desarrollo.

La importancia de analizar el contexto sociodemográfico se centra mayormente en conocer y establecer la relación entre ellas con los resultados obtenidos en la prueba ITPA-3; para esto, el estudio recolectó datos de la población que definen su sociodemografía como lo son: edad, género, nacionalidad, tiempo que vive en el país, país de residencia y tipología familiar, buscando la influencia de estos factores en las habilidades psicolingüísticas y el aprendizaje.

\section{MÉTODOS}

Se realizó un estudio exploratorio, descriptivo de corte transversal con el objetivo de dar a conocer la existencia de una relación en el desarrollo psicolingüístico de estudiantes fronterizos y transfronterizos con sus factores sociodemográficos(5).

La muestra fue una población de estudiantes de una institución educativa pública que cuenta con 1978 estudiantes divididos en cuatro sedes, distribuidas en el Municipio de Villa del Rosario, de los cuales 1735 sujetos fueron evaluados en el periodo 2017-2 y el periodo 2018-1, donde se realizó la evaluación debido a su asistencia y consentimiento firmado de sus padres, o tutor legal, desde el grado primero al grado once, exceptuando los del grado transición por la edad de los mismos.

TABLA 1. Criterios de inclusión y exclusión de la población al estudio.

\begin{tabular}{|c|c|}
\hline CRITERIOS DE INCLUSIÓN & CRITERIOS DE EXCLUSIÓN \\
\hline 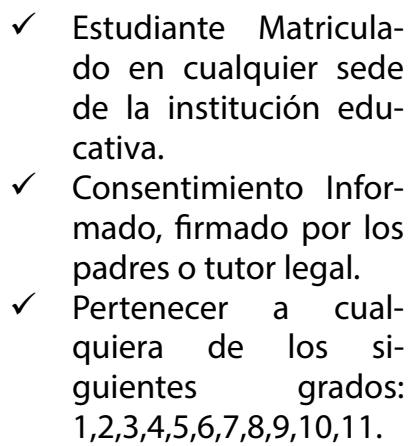 & $\begin{array}{l}\text { No estar matriculado } \\
\text { a la institución educa- } \\
\text { tiva } \\
\checkmark \quad \text { Rechazo o falta de } \\
\text { consentimiento infor- } \\
\text { mado } \\
\checkmark \quad \text { Estar cursando el gra- } \\
\text { do transición (debido } \\
\text { a la edad la prueba lo } \\
\text { excluye). }\end{array}$ \\
\hline
\end{tabular}

Fuente: Los autores

Los estudiantes fueron valorados con la prueba de habilidades psicolingüísticas Illinois en su tercera versión, ITPA-3; conformada por 12 sub pruebas, de las cuales 6 son pruebas orales y 6 escritas (1). Hacen parte de estas las siguientes subpruebas: analogías, vocabulario oral, cierre morfosintáctico, oraciones sintácticas, eliminación de sonidos, pares mínimos, secuencia de oraciones, vocabulario escrito, decodificación Visual, codificación de sonidos, ortografía visual y ortografía de sonidos. Como de 8 especificas semántica, morfosintaxis, fonética, comprensión, identificación de Palabras, ortografía, procesamiento visión símbolo y procesamiento sonido símbolo.

Para organizar la información de cada escolar, se tuvo en cuenta una serie de datos como sus factores sociodemográficos(6), para ello se analizó el género (masculino o femenino), la edad (4 a 19 años de edad), la nacionalidad (Colombiana, Venezolana u otra), país de residencia (Colombia o Venezuela), tiempo que vive en el país (menos de un año a 19 años) (7) y la tipología de estructura familiar (nuclear, amplia extenso, amplia compuesto, familiar sin núcleo, unipersonal, no familiar sin núcleo) definida por el DNP (Departamento Nacional de planificación) (8).

En la recolección de los datos de la prueba, se utilizó un apoyo virtual, que consiste en un formulario de Excel con fórmulas, que permiten la recolección de los resultados completos de la evaluación junto a las cartillas físicas del mismo y los factores sociodemográficos mencionados anteriormente, esta herramienta fue diseñada con la finalidad de realizar un registro seguro y ágil de la información con un

Revista Científica Signos Fónicos, 2019,5(2): 1-17. ISNN 2422-1716. 
fácil acceso.

Al completar y revisar la base de datos, recolectada con el Excel, se llevó a cabo el uso de un software estadístico SPSS V.21, tomando cada factor sociodemográfico y resultado de la prueba ITPA-3(9), agrupando la información en variables, para realizar un análisis de frecuencias de las mismas, para obtener de manera detallada y categorizada los datos encontrados en la primera fase del proyecto (10).

Las variables encontradas, pasaron a un instrumento de análisis $R$ (Lenguaje de Programación, The $R$ Project for Statistical Computing), que realiza con un enfoque al análisis estadístico, con un método multivariado de clasificación, basado en la exploración (clúster) (11), que permite, de manera inequívoca, mostrar la relación entre las variables o en algunos casos, muestras atípicas en la información recolectada por cada escolar.

\section{RESULTADOS}

El siguiente aspecto, trata la información recolectada de los datos sociodemográficos de los escolares evaluados en 2017-2 y 2018-1. (Edad, género, nacionalidad, país de residencia, tiempo que vive en el país, número de personas que viven en el hogar, tipología de estructura familiar,), así como también los resultados obtenidos por los escolares en cada una de las subpruebas, organizadas estas a nivel global, específico y por subpruebas.

\section{Resultados de datos sociodemográficos (variables independiente)}

TABLA 2 Género de los escolares.

\begin{tabular}{lc}
\hline & GÉNERO \\
\hline HOMBRE & $52,3 \%$ \\
\hline MUJER & $47,7 \%$ \\
\hline Total & $100 \%$ \\
\hline
\end{tabular}

En la tabla 1, se muestra la cantidad de población dividida por género, evidenciando la que existe mayor población del género masculino con un $52,3 \%$ y un $47,7 \%$ de género femenino, del total de la población evaluada en los periodos 2017-2 y 2018-1.

TABLA 3. Grado en donde se encuentran los escolares.

\begin{tabular}{cc}
\hline \multicolumn{2}{c}{ GRADO } \\
\hline 1 & $14,3 \%$ \\
\hline 2 & $11,1 \%$ \\
\hline 3 & $9,5 \%$ \\
\hline 4 & $9,6 \%$ \\
\hline 5 & $10,4 \%$ \\
\hline 6 & $8,9 \%$ \\
\hline 7 & $10,1 \%$ \\
\hline 8 & $7,8 \%$ \\
\hline 9 & $9,6 \%$ \\
\hline 10 & $4,8 \%$ \\
\hline 11 & $4 \%$ \\
\hline
\end{tabular}

Revista Científica Signos Fónicos, 2019,5(2): 1-17. ISNN 2422-1716. 


\begin{tabular}{cc}
\hline Total & $100 \%$ \\
\hline \multicolumn{3}{c}{ Fuente: los autores. }
\end{tabular}

Se observa el número de escolares evaluados por grado, encontrando mayor cantidad de población en el grado primero con un 14,3\% y un menor número de población en el grado 11 con un $4 \%$ del total de la muestra evaluada.

TABLA 3. Rango de edad por intervalos.

\begin{tabular}{lc}
\hline \multicolumn{2}{c}{ RANGO DE EDAD } \\
\hline 4 - 9 AÑOS & $37,8 \%$ \\
\hline $10-14$ AÑOS & $42,7 \%$ \\
\hline $15-19$ AÑOS & $18,8 \%$ \\
\hline Total & $100 \%$ \\
\hline
\end{tabular}

Esta representación contiene el porcentaje perteneciente a los diferentes rangos de edad evaluados en la prueba, el rango con mayor población fue de 10 a 14 años de edad con $42,7 \%$, de 4 a $937,8 \%$ y 15 a 19 años con la menor población con 18,8\%

TABLA 4. Nacionalidad de los escolares.

\begin{tabular}{ll}
\hline & NACIONALIDAD \\
\hline COLOMBIANO & $72,4 \%$ \\
\hline OTROS & $0,1 \%$ \\
\hline VENEZOLANO & $27,5 \%$ \\
\hline Total & $100 \%$ \\
\hline
\end{tabular}

En la tabla anterior se evidencia el porcentaje de estudiantes en relación a la nacionalidad obteniendo mayor cantidad de colombianos con un $72,4 \%$ y los venezolanos representan un $27,5 \%$.

TABLA 5. País de residencia de los escolares.

\begin{tabular}{lc}
\hline & PAÍS DE RESIDENCIA \\
\hline COLOMBIA & $96,6 \%$ \\
\hline VENEZUELA & $3,4 \%$ \\
\hline Total & $100 \%$ \\
\hline
\end{tabular}

Se observa el porcentaje de estudiantes en relación al país de residencia, evidenciando un mayor porcentaje de residentes en Colombia con un $96,6 \%$ y un $3,4 \%$ son residentes en Venezuela.

TABLA 6. Tiempo de vida en el país por intervalos.

\begin{tabular}{ll}
\hline \multicolumn{2}{c}{ RANGO DE TIEMPO QUE VIVE EN EL PAÍS } \\
\hline 1 MES- 11 MESES & $25,0 \%$ \\
\hline 1 AÑO - 4 AÑOS Y 11 MESES & $5,0 \%$ \\
\hline 5 AÑOS - 8 AÑOS Y 11 MESES & $27,1 \%$ \\
\hline 9 AÑOS - 12 AÑOSY 11 MESES & $31,5 \%$ \\
\hline
\end{tabular}




\begin{tabular}{ll}
\hline 13 AÑOS - 16 AÑOS Y 11 MESES & $0,1 \%$ \\
\hline 17 AÑOS - 19 AÑOSY 11 MESES & $4,5 \%$ \\
\hline NO REPORTA & $0,3 \%$ \\
\hline NUNCA HAN VIVIDO EN COLOMBIA & $2,3 \%$ \\
\hline Total & $100 \%$ \\
\hline \multicolumn{2}{c}{ Fuente: los autores. }
\end{tabular}

Se aprecia el porcentaje en cuanto al rango de tiempo que los estudiantes han vivido en el país, evidenciando un mayor porcentaje entre los 9 años - 12 años y meses con un $31,5 \%$.

TABLA 7. Número de personas que conforman el hogar del escolar.

\begin{tabular}{cc}
\hline NÚMERO DE PERSONAS EN EL HOGAR \\
\hline 0 & $0,4 \%$ \\
\hline 1 & $0,7 \%$ \\
\hline 2 & $5,2 \%$ \\
\hline 3 & $14,5 \%$ \\
\hline 4 & $28,8 \%$ \\
\hline 5 & $23 \%$ \\
\hline 6 & $11,1 \%$ \\
\hline 7 & $6,9 \%$ \\
\hline 8 & $4,1 \%$ \\
\hline 9 & $1,9 \%$ \\
\hline 10 & $1,7 \%$ \\
\hline 11 & $0,5 \%$ \\
\hline 12 & $0,6 \%$ \\
\hline 13 & $0,2 \%$ \\
\hline 14 & $0,2 \%$ \\
\hline 15 & $0,1 \%$ \\
\hline 18 & $0,1 \%$ \\
\hline 20 & $0,1 \%$ \\
\hline Total & $100 \%$ \\
\hline & Fuente: los autores. \\
\hline
\end{tabular}

En la tabla № 9 se evidencia el porcentaje en relación al número de personas que viven el hogar siendo en mayor cantidad 4 personas por hogar y dicho valor se reflejan en un $28,8 \%$.

TABLA 8. Tipo de estructura familiar que posee el escolar.

\begin{tabular}{cc}
\hline \multicolumn{2}{c}{ TIPOLOGÍA DE ESTRUCTURA FAMILIAR } \\
\hline AMPLIO COMPUESTO & $6,4 \%$ \\
\hline AMPLIO EXTENSO & $31,9 \%$ \\
\hline FAMILIA NUCLEAR & $57,2 \%$ \\
\hline FAMILIAR SIN NÚCLEO & $2,8 \%$ \\
\hline NO FAMILIAR SIN NÚCLEO & $0,1 \%$ \\
\hline NO REPORTA & $0,4 \%$ \\
\hline
\end{tabular}




\begin{tabular}{cc}
\hline UNIPERSONAL & $1,2 \%$ \\
\hline Total & $100 \%$ \\
\hline \multicolumn{2}{c}{ Fuente: los autores. }
\end{tabular}

Se aprecia el porcentaje en relación a la tipología de la estructura familiar, evidenciando mayor prevalencia de estructura familiar Amplio extenso con un 57,2\% y en menor prevalencia la estructura No familiar sin núcleo con un $0,1 \%$.

\section{Resultados de la prueba itpa-3 (variables dependientes)}

En cuanto a los resultados de la evaluación con el instrumento ITPA-3, se encuentran organizados por componentes global, específicos y sub pruebas (orales y escritos).

\section{Componente global}

TABLA 9. Resultados de los tres componentes globales de la prueba ITPA- 3(lingüístico, lenguaje oral y lenguaje escrito).

\begin{tabular}{cccc}
\hline ÍNDICES DESCRIPTIVOS & $\begin{array}{c}\text { COMPONENTE GLO- } \\
\text { BAL LINGÜÍSTICO }\end{array}$ & $\begin{array}{c}\text { COMPONENTE } \\
\text { GLOBAL DEL } \\
\text { LENGUAJE } \\
\text { ORAL }\end{array}$ & $\begin{array}{c}\text { COMPONENTE } \\
\text { GLOBAL DEL } \\
\text { LENGUAJE ES- } \\
\text { CRITO }\end{array}$ \\
\hline MUY SUPERIOR & $2,9 \%$ & $4,9 \%$ & $2,4 \%$ \\
\hline SUPERIOR & $14,2 \%$ & $10,8 \%$ & $18,6 \%$ \\
\hline ENCIMA DEL PROMEDIO & $23,0 \%$ & $15 \%$ & $31,9 \%$ \\
\hline PROMEDIO & $40,9 \%$ & $39,5 \%$ & $28,8 \%$ \\
\hline DEBAJO DEL PROMEDIO & $6,6 \%$ & $12,2 \%$ & $2,4 \%$ \\
\hline DEFICIENTE & $4,7 \%$ & $9,5 \%$ & $2,8 \%$ \\
\hline MUY DEFICIENTE & $7,4 \%$ & $8 \%$ & $6,3 \%$ \\
\hline NO APLICA & $0,4 \%$ & $0 \%$ & $6,9 \%$ \\
\hline TOTAL & $100 \%$ & $100 \%$ & $100 \%$ \\
\hline
\end{tabular}

Se puede evidenciar que, a nivel de componente global lingüístico, el mayor porcentaje de los escolares evaluados se encuentran en promedio con un $40,9 \%$, incluyendo al componente global del lenguaje oral, con un resultado del $39,5 \%$, en contraste con el componente global del lenguaje escrito, el índice por encima del promedio arrojó un 31,9\% siendo este el porcentaje más alto.

\section{Subpruebas orales}

Tabla 10. Resultados de las subpruebas orales ITPA- 3(analogías, vocabulario oral, cierre morfosintáctico, oraciones sintácticas, eliminación de sonidos pares mínimos.

\begin{tabular}{lllllll}
\hline $\begin{array}{c}\text { ÍNDICES } \\
\text { DESCRIPTIVOS }\end{array}$ & $\begin{array}{l}\text { ANALO- } \\
\text { GÍA }\end{array}$ & $\begin{array}{c}\text { VOCABULA- } \\
\text { RIO ORAL }\end{array}$ & $\begin{array}{l}\text { CIERRE MOR- } \\
\text { FOSINTÁC- } \\
\text { TICO }\end{array}$ & $\begin{array}{c}\text { ORA- } \\
\text { CIONES } \\
\text { SINTÁC- } \\
\text { TICAS }\end{array}$ & $\begin{array}{c}\text { ELIMINA- } \\
\text { CIÓN DE } \\
\text { SONIDOS }\end{array}$ & $\begin{array}{c}\text { PARES } \\
\text { MíNI- } \\
\text { MOS }\end{array}$ \\
\hline $\begin{array}{l}\text { MUY SUPE- } \\
\text { RIOR }\end{array}$ & $0,6 \%$ & $0,7 \%$ & $0,5 \%$ & $0,4 \%$ & $0,1 \%$ & $0,1 \%$ \\
\hline SUPERIOR & $8,5 \%$ & $3,6 \%$ & $10,5 \%$ & $10,6 \%$ & $22,3 \%$ & $3,6 \%$ \\
\hline $\begin{array}{c}\text { ENCIMA DEL } \\
\text { PROMEDIO }\end{array}$ & $21,8 \%$ & $16,9 \%$ & $17,4 \%$ & $8,2 \%$ & $9,6 \%$ & $1,3 \%$ \\
\hline
\end{tabular}




\begin{tabular}{lllllll}
\hline PROMEDIO & $52,6 \%$ & $58,8 \%$ & $40,5 \%$ & $45,9 \%$ & $28 \%$ & $54,7 \%$ \\
\hline $\begin{array}{c}\text { DEBAJO DEL } \\
\text { PROMEDIO }\end{array}$ & $9,5 \%$ & $10,3 \%$ & $13,9 \%$ & $13 \%$ & $7,5 \%$ & $23,1 \%$ \\
\hline DEFICIENTE & $5,2 \%$ & $7 \%$ & $13 \%$ & $9,1 \%$ & $10,9 \%$ & $8,4 \%$ \\
\hline $\begin{array}{l}\text { MUY DEFI- } \\
\text { CIENTE }\end{array}$ & $1,7 \%$ & $2,4 \%$ & $3,9 \%$ & $1 \%$ & $0,6 \%$ & $1,1 \%$ \\
\hline NO APLICA & $0,1 \%$ & $0,2 \%$ & $0,2 \%$ & $11,8 \%$ & $21 \%$ & $7,9 \%$ \\
\hline TOTAL & $100 \%$ & $100 \%$ & $100 \%$ & $100 \%$ & $100 \%$ & $100 \%$ \\
\hline & & Fuente: los autores & & &
\end{tabular}

En relación a las subpruebas orales, el índice de analogías con un porcentaje de $52,6 \%$, vocabulario oral el $58,8 \%$, cierre morfosintáctico el $40,5 \%$, oraciones sintácticas el $45,9 \%$, eliminación de sonidos el $28 \%$, y por último en la subprueba de pares mínimos el $54,7 \%$, se logra percibir que el porcentaje más alto se encuentra en promedio de acuerdo a cada subprueba.

\section{Sub pruebas escritas}

Tabla 11. Resultados de las subpruebas escritas de ITPA-3, secuencia de oraciones, vocabulario escrito, decodificación visual, codificación de sonidos, ortografía visual, ortografía de sonidos.

\begin{tabular}{lllllll}
\hline $\begin{array}{l}\text { ÍNDICES } \\
\text { DESCRIPTIVOS }\end{array}$ & $\begin{array}{c}\text { SECUEN- } \\
\text { CIA DE } \\
\text { ORA- } \\
\text { CIONES }\end{array}$ & $\begin{array}{c}\text { VOCABULA- } \\
\text { RIO ESCRI- }\end{array}$ & $\begin{array}{c}\text { CODIFI- } \\
\text { CACIÓN } \\
\text { VISUAL }\end{array}$ & $\begin{array}{c}\text { CODIFICA- } \\
\text { CIÓN DE } \\
\text { SONIDOS }\end{array}$ & $\begin{array}{c}\text { ORTO- } \\
\text { GRAFÍA } \\
\text { VISUAL }\end{array}$ & $\begin{array}{c}\text { ORTO- } \\
\text { GRAFÍA } \\
\text { DE SO- } \\
\text { NIDOS }\end{array}$ \\
\hline $\begin{array}{l}\text { MUY SUPE- } \\
\text { RIOR }\end{array}$ & $7,9 \%$ & $8,2 \%$ & $8,3 \%$ & $0,6 \%$ & $0,7 \%$ & $0,4 \%$ \\
\hline SUPERIOR & $2 \%$ & $4,6 \%$ & $12,2 \%$ & $10 \%$ & $7,7 \%$ & $4 \%$ \\
\hline $\begin{array}{l}\text { ENCIMA DEL } \\
\text { PROMEDIO }\end{array}$ & $3,6 \%$ & $3 \%$ & $0,6 \%$ & $54 \%$ & $22,5 \%$ & $34,5 \%$ \\
\hline PROMEDIO & $41,5 \%$ & $62,1 \%$ & $8,4 \%$ & $19,8 \%$ & $48,6 \%$ & $45,5 \%$ \\
\hline $\begin{array}{c}\text { DEBAJO DEL } \\
\text { PROMEDIO }\end{array}$ & $15,6 \%$ & $6,2 \%$ & $2,5 \%$ & $3,3 \%$ & $6,6 \%$ & $4,1 \%$ \\
\hline DEFICIENTE & $11,5 \%$ & $6,5 \%$ & $2,9 \%$ & $3,1 \%$ & $3,7 \%$ & $2,2 \%$ \\
\hline $\begin{array}{l}\text { MUY DEFI- } \\
\text { CIENTE }\end{array}$ & $0,2 \%$ & $0,7 \%$ & $7 \%$ & $1,8 \%$ & $2,8 \%$ & $2 \%$ \\
\hline NO APLICA & $17,8 \%$ & $8,8 \%$ & $58,1 \%$ & $7,4 \%$ & $7,3 \%$ & $7,3 \%$ \\
\hline Total & $100 \%$ & $100 \%$ & $100 \%$ & $100 \%$ & $100 \%$ & $100 \%$ \\
\hline
\end{tabular}

Se observan los índices descriptivos de la subprueba secuencia de oraciones con un porcentaje de $41,5 \%$, vocabulario escrito el $62,1 \%$ siendo estos los porcentajes más altos en las variables de promedio. En contraste a la subprueba de codificación visual con un $58,1 \%$ en el índice no aplica fue el índice porcentaje más alto, en cambio a la codificación de sonidos el $54 \%$ se encuentra por encima del promedio, ortografía visual el $48,6 \%$ ortografía de sonidos el $45,5 \%$ se encuentra en el indicativo de promedio

\section{Componentes específicos}

TABLA 12. Resultados de los componentes específicos del ITPA-3, semántica, morfosintaxis, fonéticos y comprensión. 


\begin{tabular}{|c|c|c|c|c|}
\hline $\begin{array}{l}\text { ÍNDICES } \\
\text { DESCRIPTIVOS }\end{array}$ & $\begin{array}{l}\text { COMPO- } \\
\text { NENTE } \\
\text { ESPECÍFICO } \\
\text { SEMÁNTICO }\end{array}$ & $\begin{array}{l}\text { COMPONENTE } \\
\text { ESPECÍFICO } \\
\text { MORFOSINTA- } \\
\text { XIS }\end{array}$ & $\begin{array}{l}\text { COMPONEN- } \\
\text { TE ESPECÍFI- } \\
\text { CO FONÉTI- } \\
\text { COS }\end{array}$ & $\begin{array}{l}\text { COMPONENTE } \\
\text { ESPECÍFICO } \\
\text { COMPRENSIÓN }\end{array}$ \\
\hline MUY SUPERIOR & $4,6 \%$ & $6,7 \%$ & $4 \%$ & $0,9 \%$ \\
\hline SUPERIOR & $10,3 \%$ & $9 \%$ & $8,4 \%$ & $3,1 \%$ \\
\hline $\begin{array}{l}\text { ENCIMA DEL PRO- } \\
\text { MEDIO }\end{array}$ & $21,2 \%$ & $12 \%$ & $15,9 \%$ & $18,8 \%$ \\
\hline PROMEDIO & $39,4 \%$ & $33,8 \%$ & $38,4 \%$ & $41,8 \%$ \\
\hline $\begin{array}{l}\text { DEBAJO DEL PRO- } \\
\text { MEDIO }\end{array}$ & $11,5 \%$ & $17,6 \%$ & $12,6 \%$ & $13,5 \%$ \\
\hline DEFICIENTE & $6,1 \%$ & $10 \%$ & $11,4 \%$ & $6,2 \%$ \\
\hline MUY DEFICIENTE & $6,9 \%$ & $10,7 \%$ & $9,2 \%$ & $15,5 \%$ \\
\hline NO APLICA & $0 \%$ & $0,1 \%$ & $0,1 \%$ & $0,1 \%$ \\
\hline TOTAL & $100 \%$ & $100 \%$ & $100 \%$ & $100 \%$ \\
\hline
\end{tabular}

En la tabla anterior se presentan los resultados de los componentes específicos. Referente al índice semántico se encuentra en un $39,4 \%$, en el componente morfosintáctico el $33,8 \%$, el índice fonético el $38,4 \%$ y por último el componente de comprensión en un $41,8 \%$, en relación a lo anterior se da a conocer que los componentes específicos se encuentran en el rango de promedio.

Tabla 13. Resultados de los componentes específicos del ITPA-3, identificación de palabras, ortografía, procesamiento visión símbolo.

\begin{tabular}{lllll}
\hline $\begin{array}{l}\text { ÍNDICES } \\
\text { DESCRIPTIVOS }\end{array}$ & $\begin{array}{l}\text { COMPONENTE } \\
\text { ESPECÍFICO IDEN- } \\
\text { TIFICACIÓN DE } \\
\text { PALABRAS }\end{array}$ & $\begin{array}{l}\text { COMPONENTE } \\
\text { ESPECÍFICO } \\
\text { ORTOGRAFÍA }\end{array}$ & $\begin{array}{l}\text { COMPONENTE } \\
\text { ESPECÍFICO } \\
\text { PROCESAMIEN- } \\
\text { TO VISTA-SÍM- } \\
\text { BOLO }\end{array}$ & $\begin{array}{l}\text { COMPONEN- } \\
\text { TE ESPECÍFI- } \\
\text { CO PROCE- } \\
\text { SAMIENTO } \\
\text { SONIDO-SÍM- } \\
\text { BOLO }\end{array}$ \\
\hline MUY SUPERIOR & $11,8 \%$ & $2,9 \%$ & $9,2 \%$ & $4 \%$ \\
\hline SUPERIOR & $48,1 \%$ & $14,6 \%$ & $22,1 \%$ & $23,7 \%$ \\
\hline ENCIMA DEL & $15,9 \%$ & $26,8 \%$ & $30,8 \%$ & $29,6 \%$ \\
PROMEDIO & & $33,8 \%$ & $21,0 \%$ & $24,2 \%$ \\
\hline PROMEDIO & $6,9 \%$ & $6,8 \%$ & $2,2 \%$ & $4,4 \%$ \\
\hline $\begin{array}{l}\text { DEBAJO DEL } \\
\text { PROMEDIO }\end{array}$ & $2,5 \%$ & $12,7 \%$ & $12,4 \%$ & $12 \%$ \\
\hline MUY DEFICIEN- & $12,9 \%$ & $2,3 \%$ & $2,1 \%$ & $1,9 \%$ \\
\hline TE & $1,8 \%$ & $0,1 \%$ & $0,1 \%$ & $0,1 \%$ \\
\hline DEFICIENTE & $0,1 \%$ & $100 \%$ & $100 \%$ & $100 \%$ \\
\hline NO APLICA & $100 \%$ & Fuente: los autores. & & \\
\hline TOTAL & & & & \\
\hline
\end{tabular}

Con respecto a los resultados según los componentes específicos evidenciados, se da a conocer que en identificación de palabras el índice se encuentra en superior con un $48,1 \%$, en ortografía el $33,8 \%$ se encuentra en Promedio, el componente procesamiento vista - símbolo con un 30,8\% y el procesamiento sonido - símbolo con el $29,6 \%$ se clasifican por encima del promedio. 


\section{Resultados de relación de variables}

En el análisis de configuración de redes se identificaron las relaciones entre variables, así como la conexión que presentan entre sí, a continuación, se observan los resultados dados por este análisis definiendo dos clasificaciones, por individuos y por variables de elementos en común (Clúster) (11).

\section{Resultados por grupo de individuos}

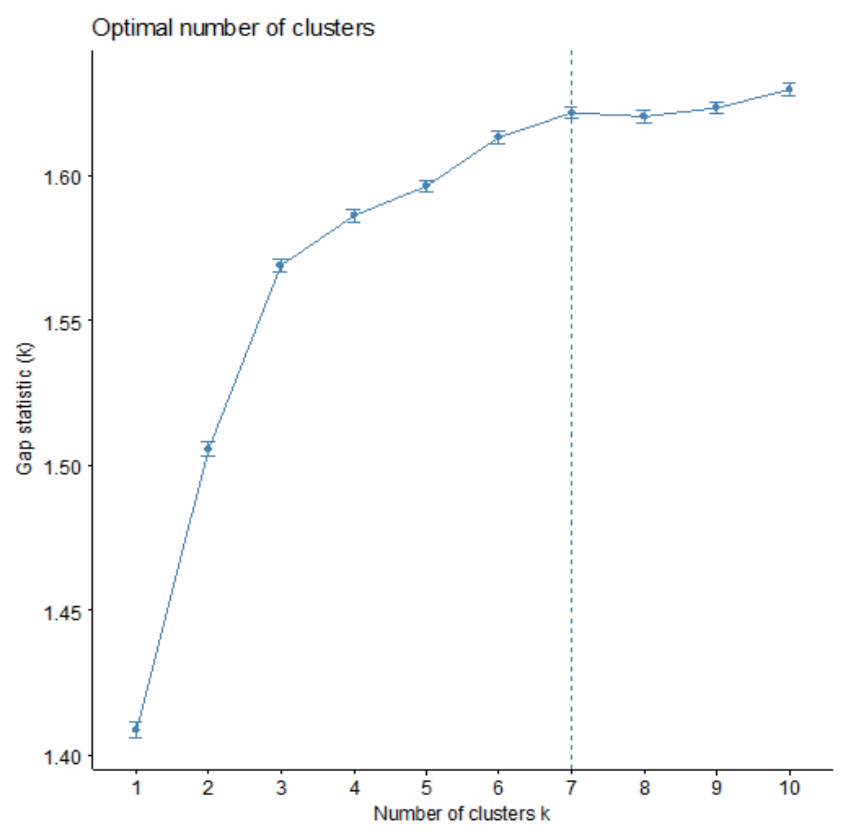

Figura 1. Número Óptimo de Clusters (Grupos). Fuente: los autores.

En la figura 1 se evidencia en el eje (x) el número de clúster conformados, la línea discontinua identifica el número de clúster homogéneos que son los que agrupan la muestra de total de 1735 individuos; cada uno delos 7 clústerestá conformado por una cantidad deindividuosque presentan características semejantes; en el eje (y) serepresenta el valor medio delas diferencias quehayentretodoslosindividuos decadagrupo.

TABLA 14. Número óptimo de clúster (Grupos).

\begin{tabular}{ccc}
\hline CLUSTER & SIZE & AVE.SIL.WIDTH \\
\hline 1 & 149 & $0,09 \%$ \\
\hline 2 & 54 & $0,22 \%$ \\
\hline 3 & 306 & $0,03 \%$ \\
\hline 4 & 145 & $0,34 \%$ \\
\hline 5 & 399 & $0,22 \%$ \\
\hline 6 & 376 & $0,11 \%$ \\
\hline 7 & 293 & $0,09 \%$ \\
\hline & Fuente: los autores.
\end{tabular}

En la tabla 14, se evidencian los clúster organizados por cantidad de individuos y la cercanía interna entre ellos; observando que los clúster con mayor concentración de individuos son el clúster 5 y 6 , es decir, si se pretende sacar una muestra representativa para aplicar un tipo de intervención, basta con escoger la muestra de uno de estos dos clúster. Los clúster 4 y 5 presentan mayor semejanza, en cuanto a las características analizadas. 


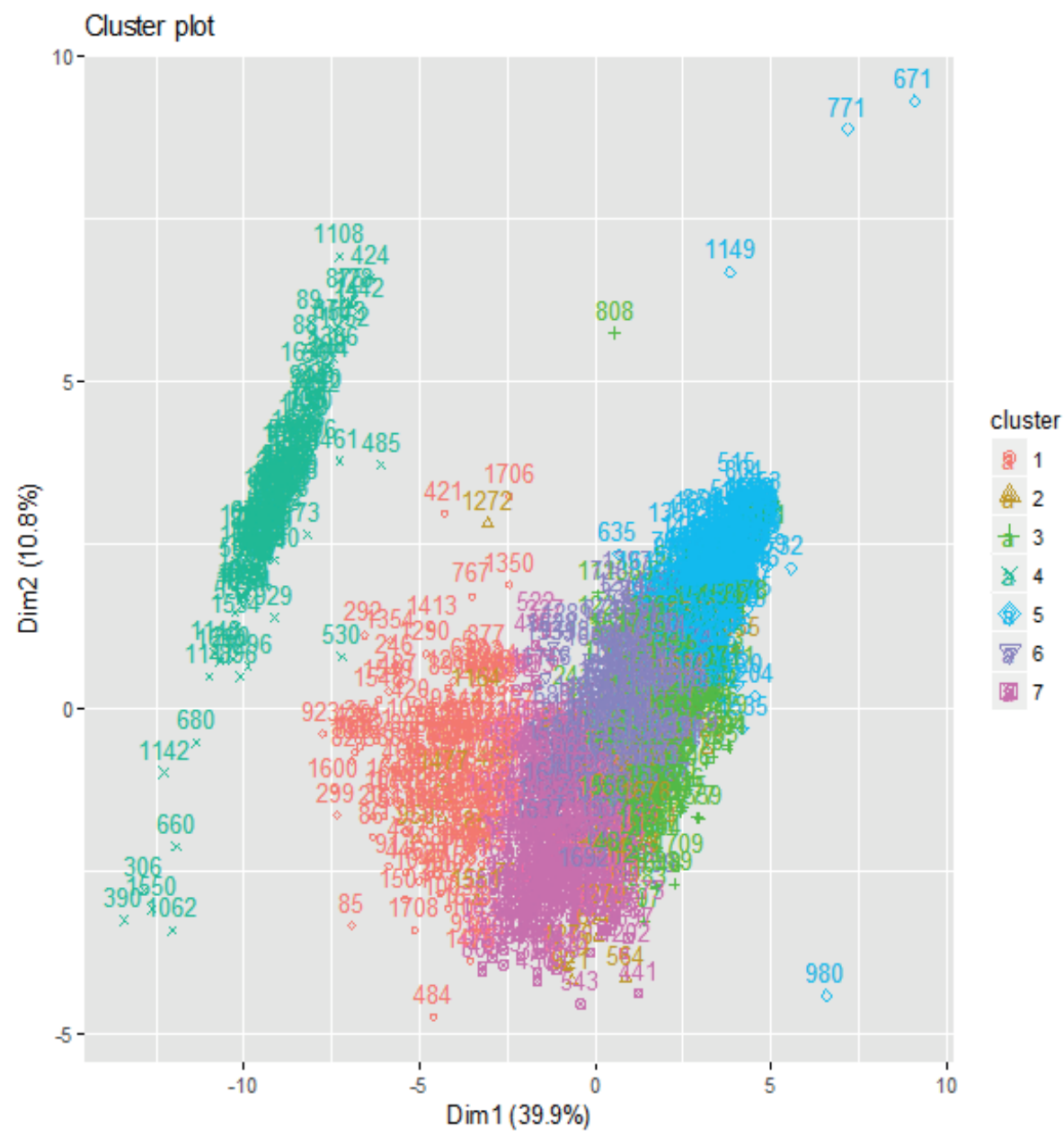

Figura 2. Configuración de los Clústeres (Individuos). Fuente: los autores.

De los 7 clúster identificados, se muestran 3 concentraciones en la figura 2. El primero está al margen izquierdo y ubica a las personas identificadas en el clúster 4 . El segundo es el de la parte central que contiene el demás clúster $(1,2,3,5,6,7)$ y el tercero se refiere a los datos atípicos de cada clúster. Esto permite ver la dispersión de las personas ubicadas en cada clúster y las características en común que comparten con el demás clúster.

\section{Resultados por grupo de variables}




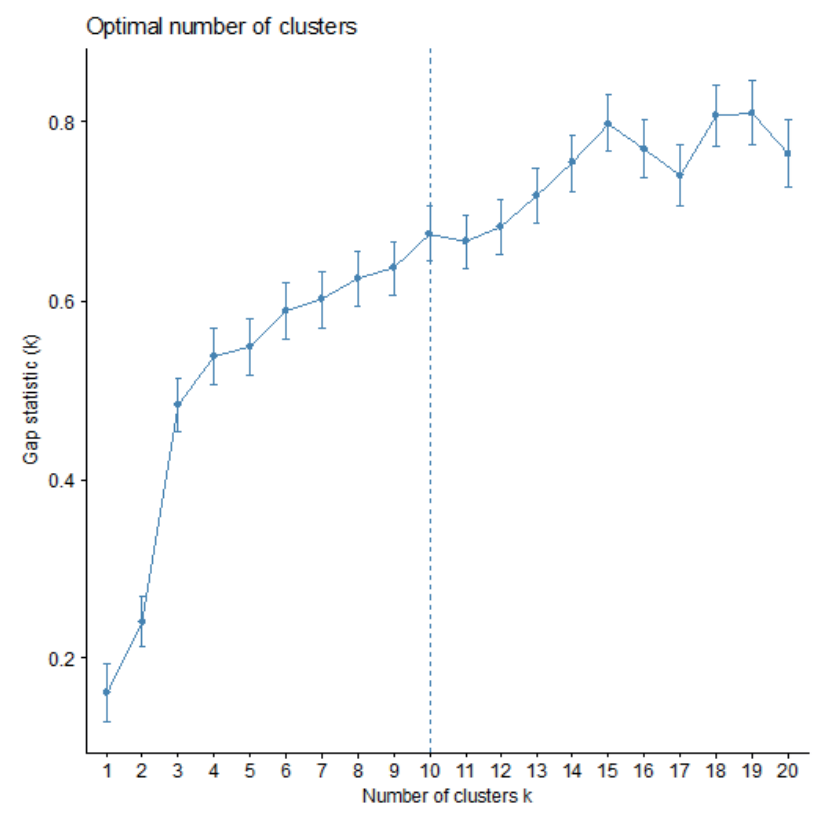

Figura 3. Clúster por variables. Fuente: los autores.

En esta figura se identifican en el eje (x), 20 clúster correspondientes a los grupos de variables, definidos por la variabilidad y la relación que hay entre ellos, la línea discontinua representa el límite óptimo de clúster pues como se observa en el clúster 11 la secuencia lineal desciende; por ello, se conforman 10 clúster con las mismas condiciones o características similares a nivel interno, en el eje (y) se representa el nivel de relación de variables; es decir entre más alta sea la puntuación, existirá mayor nivel de relación.

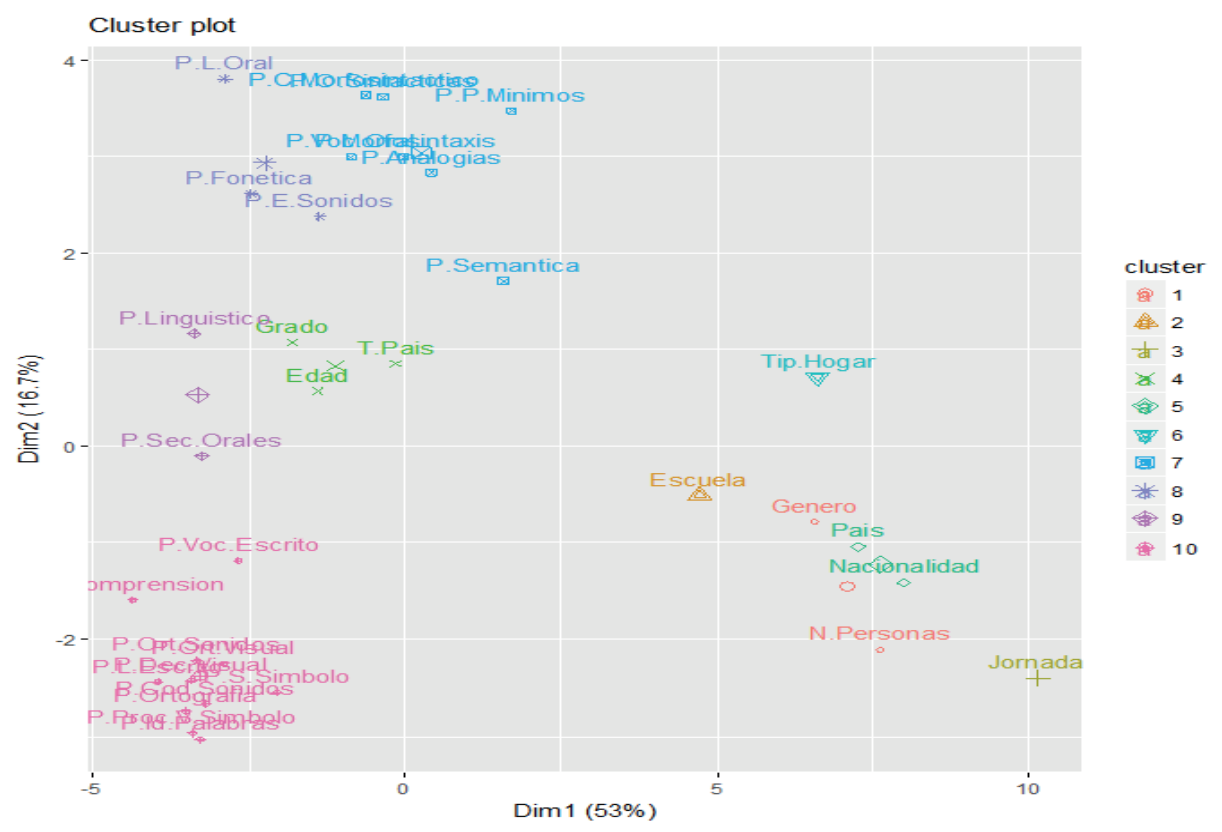

Figura 4. Clúster por variables. Fuente: los autores.

Se observa la conformación de 4 grupos que contienen los 10 clúster identificados. El primero se encuentra en el margen izquierdo inferior el cual es opuesto al segundo grupo identificado en el mismo margen, pero en su parte superior. El tercer grupo se encuentra en el margen izquierdo, pero en la parte central y el cuarto grupo se encuentra en el margen derecho inferior. Éste último es opuesto a 
los grupos ubicados en el margen izquierdo. Hay una tendencia a ubicar los grupos al margen izquierdo, lo cual significa que los datos tienen un comportamiento similar, pero tienen características diferentes al discriminarse en tres grupos; cada color indica la relación de variables entre sí, las que se observan con algún tipo de relación son:T país, Grado y edad con las puntuaciones de secuencias orales, lenguaje oral, lingüístico, eliminación de sonidos y fonética.

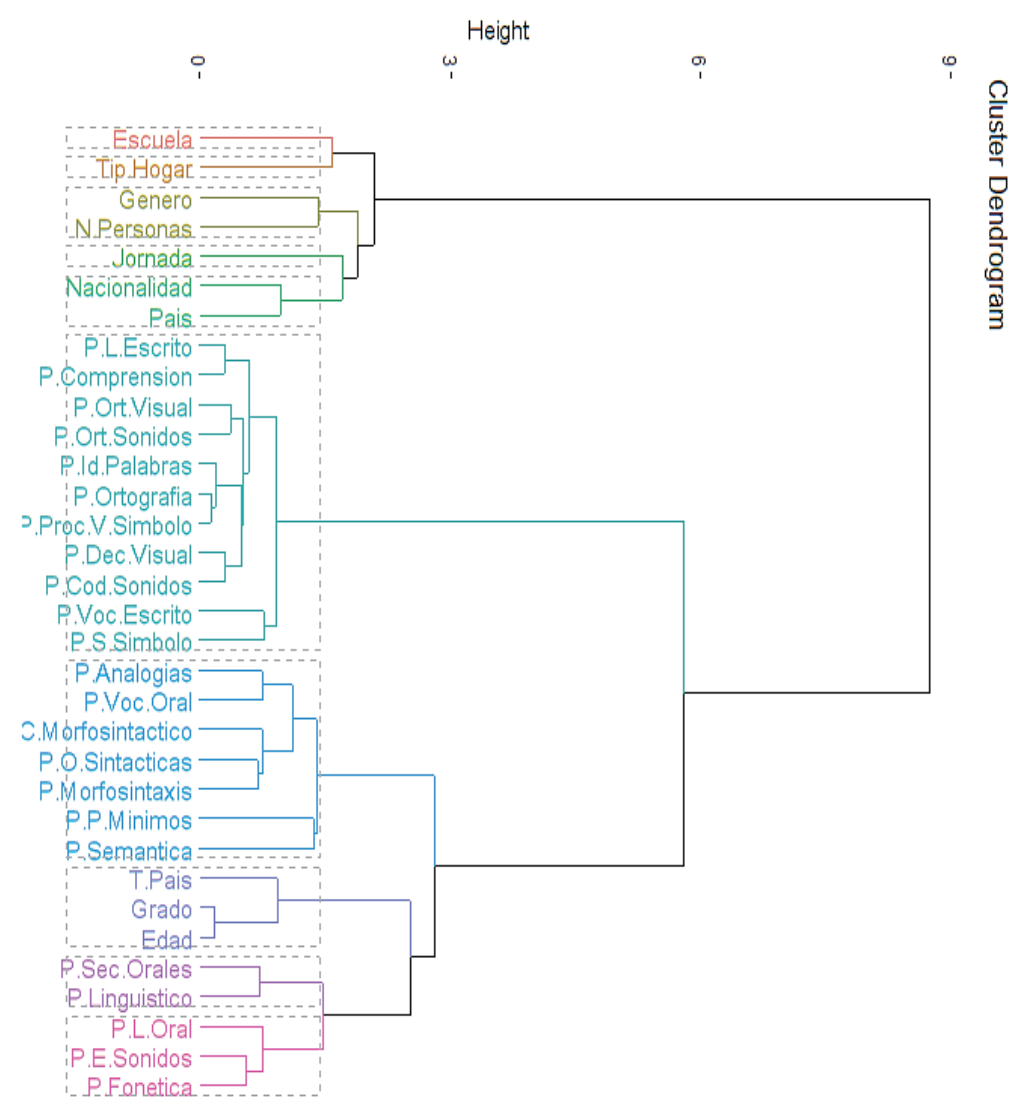

Figura 5. Nivel de agrupamiento del clúster. Fuente: los autores.

Con respecto a la figura 5 encontramos 4 grupos de los 10 clúster identificados, los cuáles son las variables compuestas en los 4 grupos generales y los subgrupos que se conforman en ellos, arrojando una influencia entre las variables de carácter poblacional T. país, grado, edad, con las sub pruebas $p$. fonética, P. Sonidos, P.L oral, P. sec orales, P. lingüístico.

\section{ANÁLISIS Y DISCUSIÓN}

Los factores sociodemográficos pueden definir parámetros importantes en el desarrollo de los escolares, al cambiar un factor, ya sea el género, nacionalidad, tipología de estructura familiar, tiempo de vivencia en un país o grado, influyen en la vida del infante, estos factores exteriorizan un compuesto de interacciones, variables y situaciones, imprescindibles en el estudio para comprender, cómo participan en el entorno de los escolares.

De modo que se considera el colegio y los índices sociodemográficos, como contextos esenciales para el desarrollo de los individuos bajo su influencia. Cualquier conjunto de individuos, que compartan un mismo contexto e interactúen con cierta frecuencia y permanencia en el espacio y el tiempo, tienden a generar características y pautas diferenciadas de funcionamiento que lo distinguen de los demás (12).

Gracias a los resultados del programa $\mathrm{R}$ (The $\mathrm{R}$ Project for Statistical Computing) se interpretan con rigor científico cada uno del clúster $(1,2,3,5,6,7)$, se evidencia que los factores determinan una relación con los procesos psicolingüísticos. Estos se encuentran asociados a los valores obtenidos de las pruebas ITPA-3. Datos que reflejan, similitud entre las características específicas de género y tipología de estructura familiar, evidenciando un nivel promedio en las habilidades psicolingüísticas 
en escolares de procedencia colombiana.

El contexto familiar, es un factor determinante en el desarrollo social de los niños, ya que es el primer escenario donde los infantes interactúan. La familia comprendida como un escenario en el que los niños y niñas aprenden sus distintas creencias, culturas y normas determinan su vinculación e integración a la sociedad, permitiendo el desarrollo personal que incide en el proceso educativo(13).

Antecedentes investigativos demuestran la importancia del contexto familiar en el desarrollo de las habilidades psicolingüísticas. En diferentes países, se ha evidenciado pruebas contundentes de que las características de las interacciones con los padres, influyen en los niveles de desarrollo del niño (14).

A su vez, la interpretación conceptual y estadística del clúster 4, permite identificar que esta agrupación de sujetos presenta las siguientes características; escolares de sexo femenino de nacionalidad venezolana, que, en los resultados encontrados en el marco de esta investigación, se especifican que un porcentaje significativo de los escolares tienen un tiempo menor a un año en el país (desde un mes a once meses).

En los resultados de la prueba ITPA-3 se evidenció, que este grupo; escolares de género femenino de nacionalidad venezolana, obtuvieron un rendimiento en un nivel promedio(15). Se identifica que, en algunos casos, el acceso a la educación en territorio colombiano puede generar problemas de adaptación; cambio del entorno, costumbres, códigos culturales, prácticas sociales y creencias, afectando sus procesos de formación académica. El anterior contexto permite identificar que las habilidades psicolingüísticas del clúster 4 son inferiores en comparación de los escolares colombianos.

Otra perspectiva de interpretación de un grupo específico, se reconoce en la gráfica Clúster Plot como casos atípicos. Los casos atípicos se caracterizan en las siguientes variables; 10 escolares de sexo femenino y nacionalidad venezolana que, al relacionarlos con las habilidades psicolingüísticas, se localizan en un nivel superior en los componentes lingüísticos, orales y escritos. A su vez, 7 escolares de sexo masculino de ambas nacionalidades se localizan en un nivel promedio en los componentes lingüísticos, orales y escritos.

En cuanto a la relación que existe entre el género y los resultados de la prueba ITPA-3, se afirma que hay un dominio en las habilidades psicolingüísticas sobre un nivel superior, correspondientes al género femenino, por otra parte, los sujetos de género masculino, obtienen un nivel promedio en las mismas. Por lo que se confirma un dato muy reiterado en educación: que las mujeres obtienen mejores resultados que los hombres en las habilidades del lenguaje(16).

Un cierto número de pruebas sugieren que, por término medio, las mujeres tienen una complejidad de habilidades que requieren el uso del lenguaje, como la fluidez verbal, la velocidad de articulación y la gramática. Suelen ser más ágiles en tareas que incluyen velocidad perceptiva y precisión manual. Por su parte, los hombres, muestran una mejor ejecución en tareas de índole espacial, entre las que se incluyen resolución de laberintos, el ensamblaje de imágenes, el dibujo de bloques, la rotación mental y las destrezas mecánicas (17).

Existe una diferencia en el cableado cerebral, lo cual conlleva a que unos y otras, desarrollen algunas tareas de manera diferente. Algunas de las diferencias que se plantean entre ambos géneros son las siguientes: Las mujeres manejan más ágilmente las tareas lingüísticas y adquieren el lenguaje a más temprana edad, presentan un nivel más eficiente en pruebas en las que tengan que generar ideas, dadas al detalle y poco pragmáticas, exhiben una ejecución superior en la recuperación de datos de la memoria.

Los hombres, por su parte responden más fácilmente al razonamiento matemático. Distinguen con mayor facilidad fondo y figura, razón por la que son mejores en situaciones de razonamiento geométrico. Mejor ejecución en el mantenimiento y manipulación de una representación mental. Son hábiles para el razonamiento abstracto, la orientación espacial e interpretación de mapas (17).

Al analizar los resultados por grupos de variables se encuentra una relación entre los factores de tiempo que vive en el país, edad y grado con las puntuaciones obtenidas en las pruebas: lingüística, lenguaje oral, secuencia de oraciones, eliminación de sonidos y fonética, analizando esta conexión se obtiene que: la variable de tiempo que vive en el país, presenta un porcentaje de dificultad del $13,8 \%$ en los individuos que han habitado el país entre 6 a 9 años, así mismo en los resultados promedio se halla un 9,2\% en individuos que han habitado el país entre 9 y 13 años.

Un estudio realizado en España, analiza las habilidades psicolingüísticas en el alumnado inmigrante

Revista Científica Signos Fónicos, 2019,5(2): 1-17. ISNN 2422-1716. 
López L. Revista Científica Signos Fónicos, 2019,5(2): 1-17.

obteniendo que este tipo de población manifiesta un nivel de dominio lingüístico inferior correspondiente a su edad cronológica, presentando mayores dificultades en los componentes de semántica y morfosintaxis (18); el presente estudio analizó de manera general los factores sociodemográficos y los resultados de la prueba ITPA-3, reflejando la concentración de dificultades en cuanto a la relación con el tiempo que vive en el país, primando las dificultades en escolares que han habitado el país entre 6 y 9 años; definiendo así el tiempo del país no resulta siendo influyente, pues los inmigrantes presentan rangos de hábitat en el país desde 1 a 4 años. Es importante aclarar que en los estudios encontrados se analizó únicamente la población que proviene de otros países, a diferencia de este que abarcó la población habitante de Colombia y Venezuela a la vez(15).

Se afirma que quienes han llegado a estudiar a otro país diferente con edad inferior a diez años tienen mejores resultados que quienes lo hicieron después de esa edad. Todo ello está lógicamente relacionado con los propios procesos de adquisición y desarrollo de las lenguas en los individuos, cuanto antes se produzca el aprendizaje de la segunda lengua, mejores serán los resultados en el proceso de adquisición, ya que las dificultades suelen ser menores (18).

Asimismo, al analizar la edad con respecto a las puntuaciones de la prueba, se observa mayores deficiencias en edades de 5 a 9 años con un 13,6\% del total de la población en este rango y en promedio encontramos un 10,6\% concentrado en edades entre 10 a 13 años.

Los escolares con edades comprendidas entre los 6 y 8 años, son los que presentan mayores deficiencias en los componentes lingüísticos, siendo estos resultados esperados en la mayoría de casos, como parece lógico va evolucionando, evidenciándose que a menor edad menor es también la puntuación en las diferentes competencias lingüísticas, y a su vez, a mayor edad mayor es la puntuación en dichas competencias; analizándolo desde el punto en que un infante a edades menores aún se encuentra consolidando los niveles lingüísticos (18), primando estas dificultades en componentes de asociación auditiva, fonética, eliminación de sonidos y procesamiento sonido símbolo (19).

Finalizando, la variable de grado, evidencia mayor dificultad en individuos que cursan primero y segundo con un $18.6 \%$. Esta variable en la literatura evidencia que la asociación auditiva, la integración gramatical y la comprensión visual constituyen habilidades en las cuales los niños de primer grado tienden a manifestar mayores dificultades, en tanto que, en tercero, los déficits principales se hallaron en la asociación y memoria auditivas y en la integración visual (18). Para esta investigación, la relación de dificultades en los niveles de primero a cuarto, se da en habilidades lingüísticas y orales, abarcando la secuencia de oraciones, eliminación de sonidos y fonética.

\section{CONCLUSIONES}

En relación con la tercera concentración (clúster) referente a los datos atípicos, existe una semejanza con las variables del sexo, la nacionalidad, el tiempo que vive en el país y la tipología de estructura familiar.

Es necesario realizar una reestructuración de las variables sociodemográficas enfatizando en algunos factores como la escolaridad de los padres, nivel socioeconómico y laboral. Ya que los factores sociodemográficos son netamente necesarios al categorizar una población de individuos según un perfil de riesgos.

Los resultados de toda la población estudiada proyectaron un nivel promedio en las habilidades psicolingüísticas, dando a entender que la mayor parte de estos se encuentran en parámetros de normalidad.

Los resultados del desarrollo psicolingüístico evaluado en los escolares, con la prueba ITPA-3, junto a el factor sociodemográfico de nacionalidad, están en el promedio, por el contrario, los artículos y publicaciones, referentes de la migración nos hablan de un bajo desempeño en los escolares.

La influencia entre la variable de sexo, con los casos atípicos y resultados del ITPA-3, las publicaciones afirman que está asociado a que las mujeres manejan más ágilmente las tareas lingüísticas y adquieren el lenguaje a más temprana edad.

En los escolares el desarrollo de las habilidades psicolingüísticas, se encuentran relacionadas en el tipo de estructura familiar, debido a la importancia del contexto ya que limitan o favorecen el acceso a dichas habilidades. 
López L. Revista Científica Signos Fónicos, 2019,5(2): 1-17.

La edad y el grado constituyen factores influyentes en los resultados de las subpruebas aplicadas; pues se evidencia que las diferentes habilidades se van adquiriendo en el transcurso del proceso educativo, por ello en la prueba aplicada se resalta el valor de tomar el dato de la edad con mayor precisión para evitar posibles distorsiones de la información así como también califica de manera equitativa de acuerdo a la edad del escolar; se evidencia que en los grados más bajos las dificultades se presentan en el lenguaje oral y la fonética; pues estas representan las habilidades que se adquieren al principio del proceso.

\section{REFERENCIAS BIBLIOGRÁFICAS}

1. García Rodríguez LF, Martínez Martínez A, Zuluaga Castro E. Diagnóstico de niños con TEL y dificultades de aprendizaje con la ITPA-3. Areté [Internet]. 2014;14(14):183-94. Available from: https:// dialnet.unirioja.es/descarga/articulo/5108950.pdf\%5Cnhttps://dialnet.unirioja.es/servlet/extart?codigo $=5108950$

2. Sánchez PA, Costa LC, Jiménez MSR. Influencia de un programa de desarrollo de las habilidades psicolingüísticas en el acceso a la lecto-escritura [Internet]. Vol. 20, Revista de Investigación Educativa. 2002. p. 189-208. Available from: http://revistas.um.es/rie/article/view/97611

3. CAROCA S, SEPÚLVEDA F. Habilidades Psicolingüísticas En Niños Con Trastorno Específico Del Lenguaje De Kinder Y Nivel Básico 1 (Nb1). TesisUchileCl [Internet]. 2008; Available from: http://www. tesis.uchile.cl/tesis/uchile/2008/blanco_s/sources/blanco_s.pdf

4. CEPAL U. Los datos demográficos: alcances, limitaciones y métodos de evaluación. 2014 [cited 2018 May 28];82. Available from: https://repositorio.cepal.org/bitstream/handle/11362/37145/ S1420555_es.pdf;jsessionid=214A3CA7DFD67DFBEBF49DB4A931B888? sequence=1

5. González GJ, Vega MG. Condiciones sociodemograficas y estado nutricional de niños menores de un año en areas perifericas de Guadalajara, México. Rev Saude Publica. 1994;28(4):268-76.

6. Portilla EMP, Claro DAB, Rodriguez SCP, Castro YMP, Carreño LGL, Ramirez MMR, et al. Analisis y validación metodologica para el estudio de los determinantes sociales en salud y discapacidad. Rev CIENTÍFICA SIGNOS FÓNICOS [Internet]. 2016 Apr 1 [cited 2018 May 24];2(1). Available from: http:// revistas.unipamplona.edu.co/ojs_viceinves/index.php/CDH/article/view/1838

7. Portilla EMP, Murillo EAA, Espinel DEB, Moncada LB, Parra IYM, Acevedo SSG, et al. Educación para la diversidad. Rev CIENTÍFICA SIGNOS FÓNICOS [Internet]. 2015 Oct 1 [cited 2018 May 24];1(3). Available from: http://revistas.unipamplona.edu.co/ojs_viceinves/index.php/CDH/article/view/1570

8. DNP (Departamento Nacional de, Planeación). Tipologías de Familias en Colombia: Evolución 1993 - 2014. Obs Políticas las Fam -OPF [Internet]. 2015 [cited 2018 May 28];9-15. Available from: http://observatoriodefamilia.dnp.gov.co/Portals/0/Tipologias evolucion_dic3 (2).pdf

9. Galicia M IX, Sánchez V A, Pavón F S, Peña F T. Habilidades psicolingüísticas al ingreso y egreso del jardín de niños. Rev Intercont Psicol y Educ [Internet]. [cited 2018 May 29];11(2):13-36. Available from: http://www.redalyc.org/pdf/802/80212414002.pdf

10. Gallego CAM, Parada YPJ, Nieto APA, Brito YR, Redondo AL, Ascanio J, et al. Determinantes sociales en salud: construcción de conocimeinto a traves de la cartografia social. Rev CIENTíFICA SIGNOS FÓNICOS [Internet]. 2015 Oct 1 [cited 2018 May 24];1(3). Available from: http://revistas.unipamplona. edu.co/ojs_viceinves/index.php/CDH/article/view/1888

11. Castro H LM, Escobar Y. Análisis Clúster como tecnica de análisis exploratorio de registros multiples en datos meteorologicos cluster. 2012;

12. García JV, Quiroz COA, Castro SBE. Autoevaluación del desempeño escolar y su relación con variables sociodemográficas en estudiantes de primaria de Sonora, México. Educ y Cienc (ISSN 2448525X) [Internet]. 2016 Jan 29 [cited 2018 May 28];4(44). Available from: http://www.educacionyciencia. org/index.php/educacionyciencia/article/view/325

13. Torres LE, Rodriguez NY. Enseñanza e investigación en psicología. [Internet]. Vol. 11, Enseñanza e Investigación en Psicología. Consejo Nacional para la Enseñanza e Investigación en Psicología; 2006 [cited 2018 May 28]. 255-270 p. Available from: http://www.redalyc.org/html/292/29211204/

14. Manzano Aragüez, Desireé; Alemany Arrebola I. Análisis de las habilidades linguisticas en educación infantil y primaria en un contexto multicultural. Profesorado Rev Currículum y

Revista Científica Signos Fónicos, 2019,5(2): 1-17. ISNN 2422-1716. 
Form Profr [Internet]. 2017 [cited 2018 May 28];21:309-29. Available from: http://www.redalyc.org/ pdf/567/56752038017.pdf

15. PISA. Compare su país con la OCDE, Panorama por país. [Internet]. PISA RESULTS. 2015 [cited 2018 May 26]. Available from: http://www.compareyourcountry.org/pisa/country/col?lg=es

16. Gil ÁP. Educación en función del sexo. [cited 2018 May 28]; Available from: http://biblioteca. ucm.es/revcul/e-learning-innova/29/art1332.pdf

17. Fajardo LA. Aproximación a la relación entre cerebro y lenguaje. Cuad Lingüistica Hispánica [Internet]. 2008;11:93-104. Available from: http://www.redalyc.org/pdf/3222/322227496008.pdf

18. José M, Merino G, Moreno Manso JM, Suárez Muñoz Á, Elena M, Sánchez G-B. Habilidades psicolingüísticas del alumnado inmigrante Psycholinguistic skills of immigrant pupils. Rev Psicol y Educ [Internet]. 2014 [cited 2018 May 29];9(1):85-101. Available from: http://www.revistadepsicologiayeducacion.es/pdf/103.pdf

19. José M, Merino G, Moreno Manso JM, Suárez Muñoz Á, Elena M, Sánchez G-B. Habilidades psicolingüísticas del alumnado inmigrante Psycholinguistic skills of immigrant pupils. Rev Psicol y Educ [Internet]. 2014 [cited 2018 May 28];9(1):85-101. Available from: http://www.revistadepsicologiayeducacion.es/pdf/103.pdf 